\title{
On the Application of the Honeycomb Conjecture to the Bee's Honeycomb*†
}

\author{
Tim Räz
}

July 29, 2014

\begin{abstract}
In a recent paper, Aidan Lyon and Mark Colyvan have proposed an explanation of the structure of the bee's honeycomb based on the mathematical Honeycomb Conjecture. This explanation has instantly become one of the standard examples in the philosophical debate on mathematical explanations of physical phenomena. In this critical note, I argue that the explanation is not scientifically adequate. The reason for this is that the explanation fails to do justice to the essentially three-dimensional structure of the bee's honeycomb.
\end{abstract}

\section{Contents}

\begin{tabular}{lll}
\hline 1 & Introduction & 2
\end{tabular}

2 Lyon's and Colyvan's Explanation 2

3 Baker: A Philosophical Motivation 3

4 Why the Explanation Fails 4

4.1 The Explanation is Incomplete . . . . . . . . . . . . . . . . . 4

4.2 The Honeycomb Conjecture Is (Probably) Irrelevant . . . . . 7

\footnotetext{
${ }^{*}$ Acknowledgements: I am grateful to Mark Colyvan, Matthias Egg, Michael Esfeld, Martin Gasser, Marion Hämmerli, Michael Messerli, Antoine Muller, Christian Sachse, Tilman Sauer, Raphael Scholl, two anonymous referees, and the participants of the philosophy of science research seminar in the fall of 2012 in Lausanne for discussions and comments on previous drafts of this paper. The usual disclaimer applies. This work was supported by the Swiss National Science Foundation, grants (100011-124462/1), (100018$140201 / 1)$

${ }^{\dagger}$ This is a pre-copyedited, author-produced PDF of an article accepted for publication in Philosophia Mathematica following peer review. The definitive publisher-authenticated version, Räz, T. (2013): On the Application of the Honeycomb Conjecture to the Bee's Honeycomb. Philosophia Mathematica 21(3): 351-60, is available online here.

${ }^{\ddagger}$ e-mail: tim.raez@gmail.com
} 


\section{Introduction}

The philosophical debate on mathematical explanations of physical phenomena relies on good, real-life examples of such explanations, and philosophers have considerably widened the stock of examples that serve as starting point for philosophical reflection. I think this is a positive trend.

However, our examples should better be scientifically adequate. I will argue in the present paper that one of the standard examples of the debate, the explanation of the structure of the bee's honeycomb based on the mathematical Honeycomb Conjecture, is not scientifically adequate. I will cast doubt on the idea that the Honeycomb Conjecture is part of an explanation of the structure of the bee's honeycomb - the purported explanation is flawed on mathematical grounds. I establish the importance of this result by showing that my arguments undermine a recent philosophical argument by Alan Baker (2012).

\section{Lyon's and Colyvan's Explanation}

The explanation of the geometric structure of the bee's honeycomb based on the Honeycomb Conjecture (HC) was first proposed by Aidan Lyon and Mark Colyvan in their 2008 paper "The Explanatory Power of Phase Space" 1 The explanandum is that the bee's honeycomb has a hexagonal shape as opposed to some other geometric shape. The explanans has two parts, one biological, the other mathematical. The biological part is that it is evolutionary advantageous to minimize the amount of wax used in the construction of honeycombs; Lyon and Colyvan trace this part of the explanation back to Darwin. The mathematical part of the explanation is provided by the Honeycomb Conjecture and its recent proof by Thomas Hales (2001). The Honeycomb Conjecture states that " a hexagonal grid represents the best way to divide a surface into regions of equal area with the least total perimeter" Lyon and Colyvan, 2008, pp. 228). Figure 1 shows a part of the hexagonal grid. Lyon and Colyvan claim that the combination of these facts together explains the structure of the bee's honeycomb.

This explanation of a physical phenomenon based on a mathematical theorem has subsequently been adopted as as a standard example in the philosophical discussion of mathematical explanations, see e.g. Baker (2009),

\footnotetext{
${ }^{1}$ This is not to say that Lyon and Colyvan are the first to suggest a connection between the bee's honeycomb and some mathematical conjecture. As early as 36 B.C., Marcus Terentius Varro claimed that the hexagon "encloses the greatest amount of space", which explains the structure of the bee's honeycomb, see Hales (2000, p. 448). However, Lyon and Colyvan introduced the explanation into the philosophical discussion.
} 




Figure 1: Hexagonal Tiling

Baker and Colyvan (2011), Saatsi (2011), Lyon (2012), Baker (2012), Tallant (2013) and there has been considerable disagreement about its philosophical analysis and significance.

So far, it has never been disputed that the explanation given by Lyon and Colyvan is acceptable on mathematical or scientific grounds. This is what I will to do in section 4. Now I will illustrate the importance of the case for the philosophical discussion.

\section{Baker: A Philosophical Motivation}

One might think that the honeycomb case is but one of many examples proposed and discussed by philosophers, and that therefore, while it is regrettable if it turns out not to be an actual explanation, this will not really affect philosophical arguments. However, this is not so. A recent paper by Alan Baker (2012) relies to a good part on the scientific adequacy of the honeycomb case.

In his paper, Baker attacks the so-called Transmission View of mathematical explanations in science (MES), which he attributes to Mark Steiner. According to this view, MES work via a transmission of an intra-mathematical explanation to some physical explanandum. The MES with explanans $M$, typically a proof, used in the explanation of a physical explanandum $P^{*}$, written $M \rightarrow P^{*}$ is, first and foremost, an explanation of an intra-mathematical explanandum $M^{*}$, written $M \rightarrow M^{*}$, and the explanation of $M^{*}$ is transmitted to $P^{*}$ via a bridge principle, written $M^{*} \leftrightarrow P^{*}$. If we remove the bridge principle from the complete MES, $M \rightarrow M^{*} \leftrightarrow P^{*}$, we are left with an intra-mathematical explanation, $M \rightarrow M^{*}$.

According to Baker, there are two separate problems with this view. The first is a counterexample, the honeycomb case. The second is an argument for the thesis that the proof of a mathematical theorem is not necessarily part 
of a scientific explanation, even if the theorem is used in that explanation ${ }^{2}$

Baker notes that two conditions have to hold in order for the honeycomb case to be a counterexample. First, it has to be a genuine MES, and second, the proof must not explain the theorem. Baker thinks that the honeycomb case is clearly a genuine MES. He writes that "there is not much to be said [on this condition], other than that biologists do generally take this to be the best explanation of why honeybees build their cells in the shape of hexagons, and that it clearly makes nontrivial use of mathematics" (Baker, 2012, p. 250). Baker repeats the claim that biologists take this explanation seriously later in his paper, but he does not substantiate it with references.

I will argue below that the first condition does not hold. If this is so, then Baker's main counterexample is flawed. Thus, the honeycomb case is worth our attention. It is crucial to get a scientifically adequate picture.

\section{Why the Explanation Fails}

In this section, I argue that the explanation proposed by Lyon and Colyvan is an inadequate explanation of the structure of the bee's honeycomb for mathematical reasons.

I first show that the explanation is deficient because the HC solves a two-dimensional problem, but the actual honeycomb has a three-dimensional structure that cannot be adequately captured in two dimensions. This establishes that the HC provides only a fraction of the mathematics relevant to the bee's honeycomb at best.

I then cast doubt on the idea that we should accept the HC even as a partial explanation of the hexagonal shape that is part of the actual, threedimensional honeycomb. The problem is that once we consider the honeycomb in three dimensions, the adequacy of a separate explanation of the two-dimensional substructure becomes dubious.

\subsection{The Explanation is Incomplete}

Lyon and Colyvan claim that Hales's theorem can help to explain what they call the hexagonal structure of the honeycomb. This presupposes that the structure of the honeycomb is in fact hexagonal - but this is incorrect. It is only the form of the openings of honeycombs, or their prismatic base, that show a hexagonal pattern, not the entire honeycomb. Actual honeycombs show hexagonal openings on the surface, but their actual geometric structure is more complicated than this: Honeycombs consist of two layers of congruent

\footnotetext{
${ }^{2}$ Baker does not want to rest his entire argument on just one counterexample, and notes the application of the four-color theorem as a second counterexample. I will not assess the strength of this example. However, the honeycomb case is his main case study and appears to carry most of the argumentative weight. I will not discuss Baker's second problem.
} 
cells, each one with a hexagonal opening and a non-flat bottom; see figure 2 for an approximate geometrical representation of a cell.

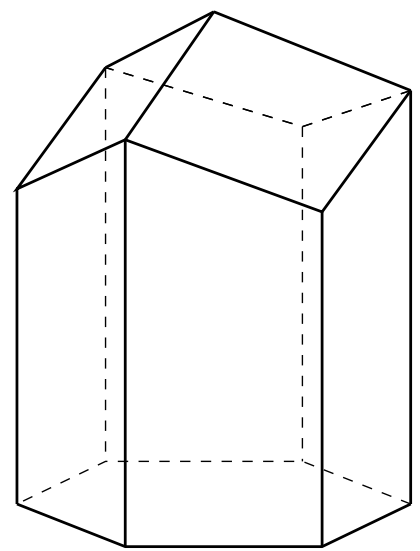

Figure 2: The three-dimensional Honeycomb

Mathematically speaking, the problem with Lyon's and Colyvan's proposal is that their explanation applies to a two-dimensional structure, whereas the actual honeycomb is three-dimensional. The HC says: "any partition of the plane into regions of equal area has perimeter at least that of the regular hexagonal honeycomb tiling"(Hales, 2001, abstract). The bee's honeycomb, however, is a three-dimensional structure that does not reduce to the two-dimensional case. What we should be looking for is an optimal threedimensional structure that can be applied to the actual honeycomb instead of the two-dimensional HC. Put simply, the structure should minimize area relative to the volume of cells instead of perimeter relative to area.

To treat the honeycomb as a two-dimensional optimization problem is not a priori unreasonable, but on closer inspection, it proves to be problematic. For example, if the honeycomb consisted of one thin layer of hexagonal cells only, then a two-dimensional description would probably capture the relevant aspects of the structure $3^{3}$ However, it is simply a fact that the honeycomb has a non-trivial three-dimensional structure. The critical point is that the actual structure comes in two layers such that the cells are open on one end only. That is why we cannot possibly account for the shape of the rhombic caps in two dimensions: the caps do not fit into the two-dimensional representation and would have to be omitted - the third dimension is necessary to represent this aspect of the structure. The three rhombi can be seen very nicely in the geometrical representation, see figure 2.

The problem I just raised has gone unnoticed in the philosophical discussion of the honeycomb case, but it is well-known in the mathematical

\footnotetext{
${ }^{3}$ This has been suggested by Erica Klarreich, see the quote below. The qualification "probably" is necessary because the claim that this structure is optimal, despite its plausibility, would have to be proven.
} 
literature. For example, Erica Klarreich discusses Hales's proof of the HC and writes:

Hales's work confirms that the hexagonal arrangement is the one that uses the smallest amount of beeswax to create a single thin layer of cells, open on each end. In an actual honeycomb, the cells in each layer are capped by three rhombic faces, forming a rhombic dodecahedron. (Klarreich, 2000, p. 157)

Klarreich at least implicitly acknowledges that the HC does not directly apply to an actual honeycomb. The three-dimensional structure to which the two-dimensional $\mathrm{HC}$ applies is a prismatic extension of the hexagonal grid, while what Klarreich calls the actual honeycomb is depicted in figure 2.

Some formulations in the mathematical literature are even more succinct. Frank Morgan discusses the $\mathrm{HC}$ in his introduction to geometric measure theory. Immediately after stating and proving the $\mathrm{HC}$, he adds the following observations under the title "The Bees' Honeycomb":

The bees actually have a more complicated, three-dimensional problem involving how the ends of the hexagonal cells are shaped to interlock with the ends of the cells on the other side. L. Fejes Tóth $[\ldots]$ showed that the bees' three-dimensional structure can be improved slightly, at least for the mathematical model with infinitely thin walls. (Morgan, 1988, pp. 166)

Morgan states that the hexagonal grid of the $\mathrm{HC}$ is not the relevant structure for the actual honeycomb, and he even mentions an alternative approach.

It could be thought that it was Thomas Hales who suggested this application of the $\mathrm{HC}$ - but this is not so. In his paper proving the $\mathrm{HC}$, Hales discusses the historical link between the conjecture and the bee's honeycomb, but he does not propose an application of the theorem along the lines of Lyon's and Colyvan's idea.

There is a gap between the philosophical and the mathematical discussion. Mathematicians think of the bee's honeycomb as three-dimensional and do not attempt to explain it via HC. Philosophers, on the other hand, have unfortunately neglected the three-dimensionality of the structure to date.

Even if we disregard the shape of the real honeycomb, there is a systematic problem with the explanation based on the HC: It applies to twodimensional surfaces and therefore can only take the shape of the openings of the cells into account. This, however, is not sufficient. It is not clear that a structure with optimally shaped openings minimizes the amount of wax. A structure can have cells with optimal openings, but some non-optimal shape 
otherwise. We cannot infer the optimality of cells from the shape of the openings. To make sure that a structure is optimal, we have to take the whole three-dimensional structure into account.

\subsection{The Honeycomb Conjecture Is (Probably) Irrelevant}

We saw in the last subsection that Lyon's and Colyvan's explanation is incomplete: It cannot capture all that is mathematically relevant about the actual honeycomb. This still leaves open the possibility that the HC is of some relevance to the actual honeycomb. After all, the openings of the cells are hexagonal, see the bottom of figure 2- so it is possible that we can apply the HC to explain the optimal shape of the openings. This would constitute a partial explanation in that the $\mathrm{HC}$ explains a part of the structure. In this subsection, I will argue that the $\mathrm{HC}$ is probably not even a partial explanation of the shape of the actual honeycomb.

The argument is not directed against the use of mathematical optimization in explanations of physical structures; some form of mathematical optimization may be relevant to the bee's honeycomb. Before I proceed, it may be helpful to clarify the role of mathematics in this kind of explanation. What is necessary for a successful explanation involving mathematical optimization?

I argued above that the relevant optimization problem is three-dimensional in the present case. What is minimized is the amount of wax relative to cells of unit volume. Then, the optimization problem has to satisfy certain boundary conditions; one of them is that each cell needs an opening of reasonable size. A possible mathematical formulation of the problem is as a bounded form of the Kelvin problem, the optimal tiling of space with cells of equal volume, with the restriction that the cells lie between two parallel planes such that each cell has an opening in one of the planes 4 Additionally, the optimization will probably have to take the thickness of the walls into account. Of course, purely mathematical considerations will not do. For example, we have to find out if and how an "optimization process" is implemented in the world: Do the bees construct the beehive from beginning to end, or is some other process involved? These issues are still debated in the biological literature. Finally, there are some constraints on possible structures due to stability $5^{5}$

The question whether any form of mathematical optimization is relevant to the bee's honeycomb is an open scientific question. For the sake of the argument, I assume that some three-dimensional optimization problem is in fact relevant, and answer the question whether, under this assumption, the

\footnotetext{
${ }^{4}$ Fejes Tóth (1964) proposed and analyzed this kind of problem.

${ }^{5}$ See Klarreich (2000) and Hales (2000) for more on the mathematical side and Pirk et al. (2004), Hepburn et al. (2007) and Bauer and Bienefeld (2013) for more on the biological side of the story.
} 
two-dimensional $\mathrm{HC}$ is relevant to the explanation as well.

We can distinguish two cases. The first possibility is that the solution to the right mathematical optimization problem does not have cells with hexagonal openings at all. In this case, the bee's honeycomb would simply not be an optimal solution, and the $\mathrm{HC}$ would be inapplicable, as the hexagonal tiling is not part of the structure. This is a real possibility: Threedimensional geometric optimization problems are notoriously hard, and optimal solutions to three-dimensional problems relevant to the bee's honeycomb are not known. To give an example, we do not know the optimal solutions to the aforementioned mathematical honeycomb structures proposed in Fejes Tóth (1964).

The second possibility is that the hexagonal grid is part of the threedimensional structure that constitutes the solution to a three-dimensional optimization problem. Even if this is the case, it is still probable that the hexagonal grid is part of the real, three-dimensional honeycomb because this whole structure is optimal in three-dimensions, and not because the grid is the optimal solution to a two-dimensional problem. This is, once more, a qualified statement, as the relevant mathematical results for threedimensional optimization problems are not known. The hexagonal grid is nothing but a geometrical structure, and the fact that it is part of a more complex structure can be unrelated to the fact that the hexagonal grid features in the HC.

What would have to be established to show the relevance of the HC to a three-dimensional structure? The HC is one possible explanation for the shape of openings of the three-dimensional honeycomb structure. However, the structure is three-dimensional, and it is probably the solution to a different, three-dimensional optimization problem which also explains the shape of the entire structure. So one way to establish the relevance of the $\mathrm{HC}$ would be to show that the reason why the three-dimensional structure has its shape subsumes the reason why a part of the structure has its shape. Or, put differently: The proof of the optimality of the three-dimensional structure would somehow have to imply the proof in the two-dimensional case, i.e. the proof of the HC. It is unclear whether such a relation between results is plausible or can be established, as we simply do not know the relevant optimality results. However, as long as we do not know whether the HC is relevant here, we should suspend our judgement about this case.

It could be objected that the hexagonal shape of the openings does not have to be a consequence of the optimality of the three-dimensional structure, because it is a biological requirement that the cells of the honeycomb have (two-dimensional) openings in the shape of the hexagonal grid. It would then be reasonable to postulate this structure as a kind of boundary condition for the optimality of the entire, three-dimensional cells.

However, if we simply postulate the hexagonal structure as a boundary condition, then the $\mathrm{HC}$ loses its explanatory power. In this case, we do not 
use the HC to explain the structure of the bee's honeycomb, but we use it in the deduction of a different result, the optimality of a three-dimensional structure. If, on the other hand, we could prove that a) the hexagonal openings are part of the optimal three-dimensional cell structure, and that $b$ ) the proof of the optimality of the three-dimensional structure really subsumes the two-dimensional, hexagonal case, the relevance of the $\mathrm{HC}$ would not have to be postulated, but it would follow from a stronger result. In this case, the $\mathrm{HC}$ would indeed be explanatory.

In this section, I have not ruled out the possibility that the $\mathrm{HC}$ is explanatorily relevant to the actual honeycomb. However, the argument shows that the relevance of the $\mathrm{HC}$ depends on optimality results in three dimensions, and as we do not yet have a clue what the optimal solution in three dimensions might be, we should abstain from such speculations at this point of mathematical progress.

\section{Conclusion and Outlook}

My goal in this paper was to argue against the original explanation of the bee's honeycomb proposed by Lyon and Colyvan. I offered two arguments for this thesis. First, I established that the original explanation is incomplete because the real honeycomb is non-trivially three-dimensional. Second, I argued that because the real structure is three-dimensional, the $\mathrm{HC}$ could be superfluous, because what matters then is a three-dimensional problem.

The main philosophical lesson we can learn from this case is that we have to be more careful in the use of examples from science, especially if we rely on our examples to be real-life cases. The discussion also has immediate consequences for the debate on mathematical explanations in science. As the original explanation based on the $\mathrm{HC}$ is not scientifically adequate, we should stop using it as a case of mathematical explanations in science and postpone the discussion of the role of mathematics for the structure of the bee's honeycomb until we have a clear, well-founded explanation of this phenomenon. As a case in point, my argument undermines the use of the honeycomb case as a counterexample by Alan Baker.

\section{References}

Baker, A. (2009). Mathematical explanation in science. British Journal for the Philosophy of Science, 60(3):611-633.

Baker, A. (2012). Science-driven mathematical explanation. Mind, 121(482):243-67.

Baker, A. and Colyvan, M. (2011). Indexing and mathematical explanation. Philosophia Mathematica, 19(3):323-34. 
Bauer, D. and Bienefeld, K. (2013). Hexagonal comb cells of honeybees are not produced via a liquid equilibrium process. Naturwissenschaften, 100:45-49.

Fejes Tóth, L. (1964). What the bees know and what they do not know. Bulletin AMS, 70:468-81.

Hales, T. C. (2000). Cannonballs and honeycombs. Notices of the AMS, $47(4): 440-9$.

Hales, T. C. (2001). The honeycomb conjecture. Discrete and Computational Geometry, 25:1-22.

Hepburn, J. R., Muerrle, T., and Radloff, S. E. (2007). The cell bases of honeybee combs. Apidologie, 38(3):268-71.

Klarreich, E. G. (2000). Foams and honeycombs. American Scientist, 88(2):152-61.

Lyon, A. (2012). Mathematical explanations of empirical facts, and mathematical realism. Australasian Journal of Philosophy, 90(3):559-78.

Lyon, A. and Colyvan, M. (2008). The explanatory power of phase spaces. Philosophia Mathematica, 16(2):227-243.

Morgan, F. (1988). Geometric Measure Theory. A Beginner's Guide. Academic Press, Burlington, San Diego, London, third edition.

Pirk, C. W. W., Hepburn, H. R., and Radloff, S. E. (2004). Honeybee combs: construction through a liquid equilibrium process? Naturwissenschaften, 91(7):350-3.

Saatsi, J. (2011). The enhanced indispensability argument: Representational versus explanatory role of mathematics in science. British Journal for the Philosophy of Science, 62:143-54.

Tallant, J. (2013). Optimus prime: paraphrasing prime number talk. Synthese, forthcoming. 